\title{
ON A CONJECTURE OF ANDREWS
}

Padiavathamma and T.Q. SUDha

\section{Department of Studies in Mathematics University of Mysore, Manasagangotri Mysore-570 006, India}

(Received July 15, 1991)

ABSTRACT. In this paper, we prove a particular case of a conjecture of Andrews on two partition functions $A_{\lambda, k, a}(n)$ and $B_{\lambda, k, a}(n)$.

KEY WORDS AND PHRASES. Partition functions. 1991 AMS SUBJECT CLASSIFICATION CODE. 11 P82.

\section{INTRODUCTION.}

For an even integer $\lambda$, let $A_{\lambda, k, a}(n)$ denote the number of partitions of $n$ into parts such that no part $\not \equiv 0(\bmod \lambda+1)$ may be repeated and no part is $\equiv 0, \pm\left(a-\frac{\lambda}{2}\right)(\lambda+1) \bmod [(2 k-\lambda+1)(\lambda+1)]$.

For an odd integer $\lambda$, let $A_{\lambda, k, a}(n)$ denote the number of partitions of $n$ into parts such that no part $\not \equiv 0\left(\bmod \frac{\lambda+1}{2}\right)$ may be repeated, no part is $\equiv \lambda+1(\bmod 2 \lambda+2)$ and no part is $\equiv 0, \pm(2 a-\lambda) \frac{\lambda+1}{2}$ $[\bmod (2 k-\lambda+1)(\lambda+1)]$.

Let $B_{\lambda, k, a}(n)$ denote the number of partitions of $n$ of the form $b_{1}+\cdots+b_{s}$ with $b_{i} \geq b_{i+1}$, no part $\not \equiv 0(\bmod \lambda+1)$ is repeated, $b_{i}-b_{i+k-1} \geq \lambda+1$ with strict inequality if $\lambda+1 / b_{i}$ and $\sum_{i=j}^{\lambda-j+1} f_{i} \leq a-j$ for $1 \leq j \leq \frac{\lambda+1}{2}$ and $f_{1}+\cdots+f_{\lambda+1} \leq a-1$ where $f_{i}$ is the number of appearances of $j$ in the partition.

Andrews [1] conjectured the following identities for $A_{\lambda, k, a}(n)$ and $B_{\lambda, k, a}(n)$.

CONJECTURE. For $\frac{\lambda}{2}<a \leq k<\lambda$,

$$
B_{\lambda, k, a}^{(n)}=A_{\lambda, k, a}(n)
$$

for $0 \leq n<\left(\begin{array}{c}k+\lambda-a+1 \\ 2\end{array}\right)+(k-\lambda+1)(\lambda+1)$, while

when $n=\left(\begin{array}{c}k+\lambda-a+1 \\ 2\end{array}\right)+(k-\lambda+1)(\lambda+1)$.

$$
B_{\lambda, k, a}(n)=A_{\lambda, k, a}(n)+1
$$

This conjecture has been verified [1] for $3 \leq \lambda \leq 7, \frac{\lambda}{2}<k \leq \min (\lambda-1,5), \frac{\lambda}{2}<a \leq k$.

In this paper we prove the case $k=a$ of the above conjecture.

\section{PROOF.}

We prove the conjecture for $k=a$ by establishing the following identities.

CASE 1. Let $\lambda$ be even. Then
(1) $B_{\lambda, k, a}(n)=A_{\lambda, k, a}{ }^{(n)}$
for $n<\left(a-\frac{\lambda}{2}\right)(\lambda+1)$
(2) $B_{\lambda, k, a}(n)=A_{\lambda, k, a}(n)$
when $n=\left(a-\frac{\lambda}{2}\right)(\lambda+1)$ 


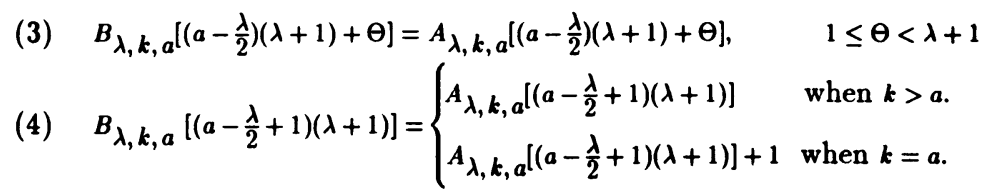

CASE 2. Let $\lambda$ be odd.

(5) $B_{\lambda, k, a}(n)=A_{\lambda, k, a}(n) \quad$ for $n \leq \lambda$.

(6) $B_{\lambda, k, a}(\lambda+1)=A_{\lambda, k, a}(\lambda+1)$

(7) $B_{\lambda, k, a}(\lambda+1+\Theta)=A_{\lambda, k, a}(\lambda+1+\Theta), \quad \Theta<\frac{\lambda+1}{2}$

(8) $\quad B_{\lambda, k, a}\left[\frac{3}{2}(\lambda+1)\right]= \begin{cases}A_{\lambda, k, a}\left[\frac{3}{2}(\lambda+1)\right], & a>\frac{\lambda+1}{2} \text { and for any } k \\ A_{\lambda, k, a}\left[\frac{3}{2}(\lambda+1)\right]+1 & \text { when } k=a=\frac{\lambda+1}{2} \text { and } k>a\end{cases}$

(9) $\quad B_{\lambda, k, a}(n)=A_{\lambda, k, a}(n), \quad n=(2 a-\lambda+1)\left(\frac{\lambda+1}{2}\right)+\Theta, \quad \Theta<\frac{\lambda+1}{2}$

(10) For $n=(2 a-\lambda+2)\left(\frac{\lambda+1}{2}\right)$

$$
B_{\lambda, k, a}(n)= \begin{cases}A_{\lambda, k, a}{ }^{(n)} & \text { when } k>a \\ A_{\lambda, k, a}(n)+1 & \text { when } k=a\end{cases}
$$

CASE 1. Let $\lambda$ be even.

PROOF OF (1). Let $P_{B_{\lambda, k, a}}$ (n) and $P_{A_{\lambda, k, a}}$ (n) denote the set of partitions enumerated by $B_{\lambda, k, a}(n)$ and $A_{\lambda, k, a}(n)$ respectively. To prove (1) we prove the following stronger result.

$$
P_{B_{\lambda, k, a}}(n)=P_{A_{\lambda, k, a}}(n) \quad \text { for } n<\left(a-\frac{\lambda}{2}\right)(\lambda+1)
$$

In fact we show that both are equal to

$$
P_{D^{(n)} \cup P_{E^{(n)}}}
$$

where $P_{D^{(n)}}$ is the set of partitions of $n$ into distinct parts and $P_{E}(n)$ is the set of partitions of $n$ in which only $(\lambda+1)$ can be repeated.

From the definition of $A_{\lambda, k, a}(n)$ it is clear that $P_{A}(n)$ is equal to (12). Also $\pi \in P_{B}(n)$ implies that $\pi \in P_{D^{(n)}}$ if $\lambda+1$ is not repeated and $\pi \in P_{E^{(n)}}$ otherwise. Hence $P_{B}(n) \subset P_{D^{(n)} \cup P_{E^{(n)}} \text {. }}$

On the other hand, let $x \in P_{D^{(n)}}$. If $n=b_{1}+\cdots+b_{k}+\cdots+b_{s}$ has more than $k$ parts, then

$$
\begin{aligned}
n & \geq 1+2+\cdots+k=1+2+\cdots+\left(\frac{\lambda}{2}+\alpha\right), \quad \text { where } k=\frac{\lambda}{2}+\alpha, \alpha<\frac{\lambda}{2} \\
& =\left(\frac{\lambda}{2}-\alpha+1+\frac{\lambda}{2}+\alpha\right)+\left(\frac{\lambda}{2}-\alpha+2+\frac{\lambda}{2}+\alpha-1\right)+\cdots+\left(\frac{\lambda}{2}+\frac{\lambda}{2}+1\right)+1+2+\cdots+\left(\frac{\lambda}{2}-\alpha\right) \\
& =(\lambda+1)+\cdots+(\lambda+1)+1+2+\cdots+\left(\frac{\lambda}{2}-\alpha\right) \\
& =\alpha(\lambda+1)+1+2+\cdots+\left(\frac{\lambda}{2}-\alpha\right)>\left(a-\frac{\lambda}{2}\right)(\lambda+1) .
\end{aligned}
$$

Thus for $n<\left(a-\frac{\lambda}{2}\right)(\lambda+1)$ and for $\pi \in P_{D^{(n)}}(n)$ no partition of $n$ contains more than $k$ parts and hence the condition on $b$ 's is satisfied.

Let us now verify the condition on $f$ 's for $\pi \in P_{D^{(n)}}$. Let $a=\frac{\lambda}{2}+\theta, \theta<\frac{\lambda}{2}$. If

$$
\sum_{i=1}^{\lambda+1} f_{i}>a-1 \quad \text { or } \quad \sum_{i=1}^{\lambda} f_{i}>a-1
$$


then the number being partitioned is

$$
\begin{aligned}
& \geq 1+2+\cdots+a=1+2+\cdots+\left(\frac{\lambda}{2}+\theta\right) \\
& =\left(\frac{\lambda}{2}-\theta+1+\frac{\lambda}{2}+\theta\right)+\left(\frac{\lambda}{2}-\theta+2+\frac{\lambda}{2}+\theta-1\right)+\cdots+\left(\frac{\lambda}{2}+\frac{\lambda}{2}+1\right)+1+2+\cdots+\left(\frac{\lambda}{2}-\theta\right) \\
& =\theta(\lambda+1)+1+2+\cdots+\left(\frac{\lambda}{2}-\theta\right)>\left(a-\frac{\lambda}{2}\right)(\lambda+1) .
\end{aligned}
$$

Thus for $n<\left(a-\frac{\lambda}{2}\right)(\lambda+1)$ and for $\pi \in P_{D^{(n)}}$, we have $\sum_{i=1}^{\lambda+1} f_{i} \leq a-1$ and $\sum_{i=1}^{\lambda} f_{i} \leq a-1$.

Similarly if $\sum_{i=2}^{\lambda} f_{i}>a-2$, then the number being partitioned is

$$
\begin{aligned}
& \geq 2+3+\cdots+\left(\frac{\lambda}{2}+\theta\right) \\
& =\theta(\lambda+1)+2+3+\cdots+\left(\frac{\lambda}{2}-\theta\right) \\
& >\left(a-\frac{\lambda}{2}\right)(\lambda+1) \quad \text { if } \frac{\lambda}{2}-\theta \geq 2 .
\end{aligned}
$$

Hence $\sum_{i=2}^{\lambda-1} f_{i} \leq a-2$ for $\frac{\lambda}{2}-\theta \geq 2$ and $n<\left(a-\frac{\lambda}{2}\right)(\lambda+1)$. Let $\frac{\lambda}{2}-\theta=1$.

Then $a=\lambda-1$ and for $\pi \in P_{D^{(n)}} . f_{i} \leq 1$ for all $i=1,2, \cdots, \lambda-1$ and hence

$$
\sum_{i=2}^{\lambda-1} f_{i} \leq \lambda-2=a-1
$$

If $\sum_{i=2}^{\lambda} f_{i}^{-1}=\lambda-2$, then the number being partitioned is

$$
\begin{aligned}
& \geq 2+3+\cdots+(\lambda-1) \\
& =(\lambda-1+2)+(\lambda-2+3)+\cdots+\left(\frac{\lambda}{2}+1+\frac{\lambda}{2}\right) \\
& =\left(\frac{\lambda}{2}-1\right)(\lambda+1)=\Theta(\lambda+1)=\left(a-\frac{\lambda}{2}\right)(\lambda+1) .
\end{aligned}
$$

Thus for $n<\left(a-\frac{\lambda}{2}\right)(\lambda+1), \sum_{i=2}^{\lambda-1} f_{i} \leq \lambda-3=a-2$.

Proceeding on the same lines we can show that the other conditions on $f$ 's are satisfied for

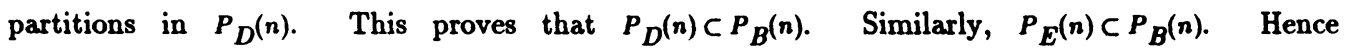
$P_{B}(n)=P_{D}(n) \cup P_{E}(n)$.

PROOF OF (2). Let $P_{A}^{\prime}(n)$ [resp. $P_{B}^{\prime}(n)$ ] denote the set of partitions enumerated by $A_{\lambda, k, a}(n)$ [resp. $\left.B_{\lambda, k, a}(n)\right]$ but not by $B_{\lambda, k, a}(n)\left[\right.$ resp. $\left.A_{\lambda, k, a}(n)\right]$. Then we claim

$$
\left.P_{A}^{\prime}(n)=[a+(a-1)+\cdots+(\lambda-a+2)+(\lambda-a+1)] \text { and } P_{B}^{\prime}(n)=\left[a-\frac{\lambda}{2}\right)(\lambda+1)\right] \text { for } n=\left(a-\frac{\lambda}{2}\right)(\lambda+1)
$$

Clearly $\pi=a+(a-1)+\cdots+(\lambda-a+1) \in P_{A}(n)$ but $\pi \notin P_{B}(n)$ as it violates the condition on $f$ 's when $j=\lambda-a+1$. In fact $f_{\lambda-a+1}+\cdots+f_{a}=a-(\lambda-a)=2 a-\lambda \leq a-(\lambda-a+1)=2 a-\lambda-1$. On the other hand, $\left(a-\frac{\lambda}{2}\right)(\lambda+1) \in P_{B}(n)$ but it does not belong to $P_{A}(n)$ since for partitions enumerated by $A_{\lambda, k, a}(n)$ no part is $\equiv\left(a-\frac{\lambda}{2}\right)(\lambda+1) \bmod [(2 k-\lambda+1)(\lambda+1)]$.

As in the proof of (1), we can show that partitions $x \neq a+(a-1)+\cdots+(\lambda-a+1) \in P_{A}(n)$ are the same as the partitions $x \neq\left(a-\frac{\lambda}{2}\right)(\lambda+1) \in P_{B}(n)$. This proves (2).

PROOF OF (3). To prove (3) we establish a bijection of $P_{A}^{\prime}(n)$ onto $P_{B}^{\prime}(n)$ where $n=\left(a-\frac{\lambda}{2}\right)(\lambda+1)+\theta, \theta<\lambda+1$. Now $\pi \in P_{A}^{\prime}(n)$ implies that it violates one of the conditions on $f$ 's or b's. Let $S_{j}\left(j=1,2, \cdots, \frac{\lambda}{2}\right)$ denote the condition

and let $S$ denote the condition

$$
\sum_{i=j}^{\lambda-j+1} f_{i} \leq a-j
$$

$$
\sum_{i=j}^{\lambda+1} f_{i} \leq a-1
$$


and let $S^{*}$ be the condition on $b^{\prime}$ 's. In the following steps 1 to $\frac{\lambda}{2}+2$ we enumerate the partitions in $P_{A}$ violating $S_{\frac{\lambda}{2}}, \cdots, S_{1}, S$ and $S^{*}$ and also give the necessary bijection of $P_{A}^{\prime}(n)$ onto $P_{B}^{\prime}(n)$.

STEP 1. Consider $S_{\frac{\lambda}{2}}: f_{\frac{\lambda}{2}}+f_{\frac{\lambda}{2}+1} \leq 2 \leq a-\frac{\lambda}{2}$. For $a-\frac{\lambda}{2} \geq 2$ there are no partitions in $P_{A}$ violating $S_{\frac{\lambda}{2}}$. If $a-\frac{\lambda}{2}=1$ then the set of partitions violating $S_{\frac{\lambda}{2}}$ is $\left\{\left(\frac{\lambda}{2}+1\right)+\frac{\lambda}{2}+\pi: \pi \in P_{D}(\theta)\right.$ with parts $\left.<\frac{\lambda}{2}\right\} \cup\left\{\left(\frac{\lambda}{2}+\Theta^{\prime}\right)+\left(\frac{\lambda}{2}+1\right)+\frac{\lambda}{2}+\pi: \pi \in P_{D}\left(\Theta-\frac{\lambda}{2}-\Theta^{\prime}\right)\right.$ with parts $\left.<\frac{\lambda}{2}, 2 \leq \Theta^{\prime} \leq \frac{\lambda}{2}\right\}$. For an element in the first set we associate $(\lambda+1)+\pi$ in $P_{B}^{\prime}$ while for an element in the second set we associate $(\lambda+1)+\left(\frac{\lambda}{2}+\Theta^{\prime}\right)+\pi$ in $P_{B}^{\prime}$.

STEP 2. Consider $S_{\frac{\lambda}{2}-1}: f_{\frac{\lambda}{2}-1}+f_{\frac{\lambda}{2}}+f_{\frac{\lambda}{2}+1}+f_{\frac{\lambda}{2}+2} \leq 4 \leq a-\frac{\lambda}{2}+1$. For $a-\frac{\lambda}{2} \geq 3$ there are no partitions in $P_{A}$ violating $S_{\frac{\lambda}{2}-1}$. Let $a-\frac{\lambda}{2}=1$. Then the set of partitions violating $S_{\frac{\lambda}{2}-1}$ is

$$
\begin{array}{ll}
\left\{\left(\frac{\lambda}{2}+1\right)+\frac{\lambda}{2}+\left(\frac{\lambda}{2}-1\right)+\pi: \pi \in P_{D}\left(\Theta-\frac{\lambda}{2}+1\right)\right. & \text { with parts } \left.<\frac{\lambda}{2}-1\right\} \\
\cup\left\{\left(\frac{\lambda}{2}+2\right)+\left(\frac{\lambda}{2}+1\right)+\frac{\lambda}{2}+\pi: \pi \in P_{D}\left(\Theta-\frac{\lambda}{2}-2\right)\right. & \text { with parts } \left.<\frac{\lambda}{2}-1\right\} \\
\cup\left\{\left(\frac{\lambda}{2}+2\right)+\frac{\lambda}{2}+\left(\frac{\lambda}{2}-1\right)+\pi: \pi \in P_{D}\left(\Theta-\frac{\lambda}{2}\right)\right. & \text { with parts } \left.<\frac{\lambda}{2}-1\right\} \\
\cup\left\{\left(\frac{\lambda}{2}+2\right)+\left(\frac{\lambda}{2}+1\right)+\left(\frac{\lambda}{2}-1\right)+\pi: \pi \in P_{D}\left(\Theta-\frac{\lambda}{2}-1\right)\right. & \text { with parts } \left.<\frac{\lambda}{2}-1\right\}
\end{array}
$$

We note that the partitions in the first two sets violate $S_{\frac{\lambda}{2}}$. For a partition in the third set we associate $(\lambda+1)+\frac{\lambda}{2}+\pi$ in $P_{B}^{\prime}$ while we associate $(\lambda+1)+\left(\frac{\lambda}{2}+1\right)+\pi$ in $P_{B}^{\prime}$ for a partition in the last set.

Let $a-\frac{\lambda}{2}=2$. The set of partitions of $2(\lambda+1)+\theta$ in $P_{A}^{\prime}$ violating $S_{\frac{\lambda}{2}-1}$ is

$$
\begin{gathered}
\left\{\left(\frac{\lambda}{2}+2\right)+\left(\frac{\lambda}{2}+1\right)+\frac{\lambda}{2}+\left(\frac{\lambda}{2}-1\right)+\pi: \pi \in P_{\left.D^{(}\right)} \quad \text { with parts }<\frac{\lambda}{2}-1\right\} \\
\cup\left\{\left(\frac{\lambda}{2}+\Theta^{\prime}\right)+\left(\frac{\lambda}{2}+2\right)+\left(\frac{\lambda}{2}+1\right)+\frac{\lambda}{2}+\left(\frac{\lambda}{2}-1\right)+\pi: \pi \in P_{D^{-}}\left(\Theta-\frac{\lambda}{2}-\Theta^{\prime}\right), \text { parts }<\frac{\lambda}{2}-1,3 \leq \Theta^{\prime} \leq \frac{\lambda}{2}\right\}
\end{gathered}
$$

For an element in the first set we associate $2(\lambda+1)+\pi$ in $P_{B}^{\prime}$ while for an element in the second set we associate $2(\lambda+1)+\left(\frac{\lambda}{2}+\Theta^{\prime}\right)+\pi$ in $P_{B}^{\prime}$. Proceeding like this we arrive at the following step.

STEP $\frac{\lambda}{2}$. Consider $S_{1}: f_{1}+\cdots+f_{\lambda} \leq a-1$. Since $f_{i} \leq 1$ for all. $i=1,2, \cdots, \lambda$ we have $f_{1}+f_{2}+\cdots+f_{\lambda} \leq \lambda$. Let $f_{1}+f_{2}+\cdots+f_{\lambda}=\lambda$. Then $1+2+\cdots+\lambda=\frac{\lambda}{2}(\lambda+1)>n$. Thus there are no partitions of $n$ in $P_{A}$ in which all parts $1,2, \cdots, \lambda$ appear. Let $f_{1}+\cdots+f_{\lambda}=\lambda-1$. Let the deleted part among $1,2, \cdots, \lambda$ be $x$. Consider

(13) $1+2+\cdots+(x-1)+(x+1)+\cdots+(\lambda-1)+\lambda=\left(\frac{\lambda}{2}-1\right)(\lambda+1)+(\lambda+1-x)$ with $1 \leq \lambda+1-x \leq \lambda$.

If $a-\frac{\lambda}{2}=\frac{\lambda}{2}-1$, then the only partition of $n$ violating $S_{1}$ is

$$
\lambda+(\lambda-1)+\cdots+(x+1)+(x-1)+\cdots+2+1
$$

with $\lambda+1-x=\theta$ for which we associate $\left(\frac{\lambda}{2}-1\right)(\lambda+1)+\theta$ in $P_{B}^{\prime}$.

When $a-\frac{\lambda}{2}<\frac{\lambda}{2}-1$, there are no partitions of $n$ violating $S_{1}$ since (13) $>n$. More generally, if $f_{1}+\cdots+f_{\lambda}=\lambda-y, 2 \leq y \leq \lambda-a$, and if $x_{1}, \cdots, x_{y}$ are the parts which are left out with $1 \leq x_{1}<x_{2}<\cdots<x_{y} \leq \lambda$, then

$$
\begin{aligned}
& \lambda+(\lambda-1)+\cdots+\left(x_{y}+1\right)+\left(x_{y}-1\right)+\cdots+\left(x_{1}+1\right)+\left(x_{1}-1\right)+\cdots+2+1 \\
& =\left(\frac{\lambda}{2}-y\right)(\lambda+1)+\left(\lambda+1-x_{1}\right)+\cdots+\left(\lambda+1-x_{y}\right)
\end{aligned}
$$


If $a-\frac{\lambda}{2}<\frac{\lambda}{2}-y$, then there are no partitions of $n$ violating $S_{1}$ since (14) $>n$. If $a-\frac{\lambda}{2}=\frac{\lambda}{2}-y$, then

$$
n=\left(a-\frac{\lambda}{2}\right)(\lambda+1)+\left(\lambda+1-x_{1}\right)+\cdots+\left(\lambda+1-x_{y}\right) .
$$

There are no partitions of $n$ violating $S_{1}$ if $\left(\lambda+1-x_{1}\right)+\cdots+\left(\lambda+1-x_{y}\right)>\theta$. The partition (14) violates $S_{1}$ when $\left(\lambda+1-x_{1}\right)+\cdots+\left(\lambda+1-x_{y}\right)=\theta$ and for this partition we associate

$$
\left(\frac{\lambda}{2}-y\right)(\lambda+1)+\left(\lambda+1-x_{1}\right)+\cdots+\left(\lambda+1-x_{y}\right) \text { in } P_{B}^{\prime} \text {. }
$$

If $\left(\lambda+1-x_{1}\right)+\cdots+\left(\lambda+1-x_{y}\right)<\theta$, then there are no partitions of $n$ violating $S_{1}$ since parts have to be repeated.

Let $a-\frac{\lambda}{2}>\frac{\lambda}{2}-y$. Then $\frac{\lambda}{2}-y+1 \leq a-\frac{\lambda}{2} \leq \frac{\lambda}{2}-1$ and there are no partitions of $n$ violating $s_{1}$ since $f_{1}+\cdots+f_{\lambda}=\lambda-y \leq a-1$.

STEP $\frac{\lambda}{2}+1$. Consider $S: f_{1}+\cdots+f_{\lambda+1} \leq a-1$. Clearly $f_{i} \leq 1$ for $i=1,2, \cdots, \lambda$ and $f_{\lambda+1} \leq a-\frac{\lambda}{2}$. Let $f_{1}+\cdots+f_{\lambda+1}=\lambda+\alpha$, where $f_{\lambda+1}=\alpha$ with $1 \leq \alpha \leq a-\frac{\lambda}{2}$. Since $1+2+\cdots+(\lambda+1)=\left(\frac{\lambda}{2}+1\right)(\lambda+1)>n$, it follows that there are no partitions of $n$ violating $S$ if $f_{1}+\cdots+f_{\lambda+1} \geq \lambda+1$. Thus let us consider the case when $f_{1}+\cdots+f_{\lambda}+f_{\lambda+1}=\lambda$ with $f_{\lambda+1}=\alpha$. Then the number being partitioned is

$$
\begin{aligned}
& \geq 1+2+\cdots+(\lambda-\alpha)+\alpha(\lambda+1) \\
& =1+2+\cdots+\alpha+\left(\frac{\lambda}{2}-\alpha\right)(\lambda+1)+\alpha(\lambda+1) \\
& =\frac{\lambda}{2}(\lambda+1)+1+2+\cdots+\alpha>n .
\end{aligned}
$$

Thus there are no partitions of $n$ violating $S$ in this case also.

More generally, let $f_{1}+\cdots+f_{\lambda+1}=\lambda-y, f_{\lambda+1}=\alpha$ with $1 \leq y \leq \lambda-a$. Let $x_{1}, \cdots, x_{y+\alpha}$ be the parts deleted among $1,2, \cdots, \lambda$ with $1 \leq x_{1}<x_{2}<\cdots<x_{y}+\alpha \leq \lambda$. Consider

$$
\begin{aligned}
& \begin{array}{r}
\underbrace{(\lambda+1)+\cdots+(\lambda+1)}_{\alpha \text { times }}+\lambda+(\lambda-1)+\cdots+\left(x_{y}+\alpha+1\right)+\left(x_{y}+\alpha-1\right)+\cdots \\
+\left(x_{1}+1\right)+\left(x_{1}-1\right)+\cdots+2+1
\end{array} \\
& =\alpha(\lambda+1)+\left(\frac{\lambda}{2}-\alpha-y\right)(\lambda+1)+\left(\lambda+1-x_{1}\right)+\cdots+\left(\lambda+1-x_{y}+\alpha\right) \\
& =\left(\frac{\lambda}{2}-y\right)(\lambda+1)+\left(\lambda+1-x_{1}\right)+\cdots+\left(\lambda+1-x_{y}+\alpha\right) \text {. }
\end{aligned}
$$

As in the case of $S_{1}$ we can show that there are no partitions of $n$ violating $S$ when $a-\frac{\lambda}{2}$ is less or greater than $\frac{\lambda}{2}-y$ and even when $a-\frac{\lambda}{2}=\frac{\lambda}{2}-y$ and $\left(\lambda+1-x_{1}\right)+\cdots+\left(\lambda+1-x_{y}+\alpha\right)$ is less or greater then $\theta$. If $\left(\lambda+1-x_{1}\right)+\cdots+\left(\lambda+1-x_{y}+\alpha\right)=\theta$ then the partition on the extreme left hand side of (15) violates $S$ for which we associate the last partition of (15) which belongs to $P_{B}^{\prime}$.

STEP $\frac{\lambda}{2}+2$. We now prove that if a partition violates the condition $S^{*}$ on $b^{\prime}$ s then it violates one of the conditions on $f$ 's. Before proving this we first note that when $k>a$ for a partition of $n=\left(a-\frac{\lambda}{2}\right)(\lambda+1)+\theta, \Theta<\lambda+1$ having $\geq k$ parts

$$
\begin{aligned}
& 1+2+\cdots+k \\
= & 1+2+\cdots+\left(\frac{\lambda}{2}+\alpha\right) \quad \text { where } k=\frac{\lambda}{2}+\alpha, 1 \leq \alpha<\frac{\lambda}{2} . \\
= & \left(\frac{\lambda}{2}+\alpha\right)+\left(\frac{\lambda}{2}-\alpha+1\right)+\cdots+\left(\frac{\lambda}{2}+1\right)+\frac{\lambda}{2}+1+2+\cdots+\left(\frac{\lambda}{2}-\alpha\right) \\
= & \left(k-\frac{\lambda}{2}\right)(\lambda+1)+1+2+\cdots+\left(\frac{\lambda}{2}-\alpha\right) \\
> & \left(a-\frac{\lambda}{2}\right)(\lambda+1)+\lambda+1>n,
\end{aligned}
$$

And hence there are no partitions of $n$ violating $S^{*}$ in this case.

Thus it suffices to consider the case when $k=a$. If a partition violates $S^{*}$ then there exists a partition 


$$
n=b_{1}+\cdots+b_{i}+\cdots+b_{i+k-1}+\cdots+b_{k}+\cdots+b_{s}
$$

and an integer $i$ with $b_{i}-b_{i+k-1}<\lambda+1$. If $b_{i+k-1} \geq \lambda+1$, then the number being partitioned is

$$
\begin{gathered}
\geq(\lambda+1)+\cdots+(\lambda+1)+\cdots \\
\geq k(\lambda+1) \geq\left(a-\frac{\lambda}{2}+1\right)(\lambda+1)>n .
\end{gathered}
$$

Thus let $b_{i+k-1}<\lambda+1$. If $b_{i}<\lambda+1$ then (16) contains at least $k$ parts $\leq \lambda$ and hence $\sum_{i=1}^{\lambda} f_{i} \geq k$ which implies that such a partition violates $S_{1}$.

Let $b_{i+k-1}<\lambda+1$ and $b_{i} \geq \lambda+1$. Since $n=\left(a-\frac{\lambda}{2}\right)(\lambda+1)+\theta, \theta<\lambda+1$, the number of parts $\geq \lambda+1$ among $b_{i}, \cdots, b_{i+k-1}$ is $\leq a-\frac{\lambda}{2}$. If $a-\frac{\lambda}{2}$ parts are equal to $\lambda+1$, then $f_{\lambda+1}=a-\frac{\lambda}{2}$ and the remaining $k-a+\frac{\lambda}{2}$ parts are $\leq \lambda$ and hence

$$
f_{1}+\cdots+f_{\lambda}+f_{\lambda+1} \geq k-a+\frac{\lambda}{2}+a-\frac{\lambda}{2}=k
$$

and such a partition violates $S$.

If a partition of a number violates $S^{*}$ and if there are parts $>\lambda+1$ then the number being partitioned is

$$
\left(\lambda+x_{\alpha}\right)+\left(\lambda+x_{\alpha-1}\right)+\cdots+\left(\lambda+x_{1}\right)+y_{1}+\cdots+y_{k-\alpha}
$$

where $\alpha<a-\frac{\lambda}{2}, 1 \leq x_{1}<x_{2}<\cdots<x_{\alpha}$ and $y_{1}, \cdots, y_{k-\alpha}$ are among $1,2, \cdots, \lambda$. Since $b_{i}-b_{i+k-1}<\lambda+1$ we have $\lambda+x_{\alpha}-y_{k-\alpha}<\lambda+1$ which implies $x_{\alpha}-y_{k-\alpha}<1$ and hence $x_{\alpha}=y_{k-\alpha}$. If $y_{k-\alpha}=x_{\alpha}>1$ then (17) is

$$
\begin{aligned}
& \geq \alpha(\lambda+1)+(k-\alpha+1)+\cdots+3+2+1 \\
& =\alpha(\lambda+1)+\left(\frac{\lambda}{2}+\beta-\alpha+1\right)+\cdots+2+1 \quad \text { where } k=\frac{\lambda}{2}+\beta, 1 \leq \beta<\frac{\lambda}{2} . \\
& =\alpha(\lambda+1)+(\beta-\alpha+1)(\lambda+1)+1+2+\cdots+\left(\frac{\lambda}{2}-\beta+\alpha-1\right) \\
& =(\beta+1)(\lambda+1)+1+2+\cdots+\left(\frac{\lambda}{2}-\beta+\alpha-1\right) \\
& =\left(k-\frac{\lambda}{2}+1\right)(\lambda+1)+1+2+\cdots+\left(\frac{\lambda}{2}-\beta+\alpha-1\right)>n .
\end{aligned}
$$

From this it is clear that if a partition of $\left(a-\frac{\lambda}{2}\right)(\lambda+1)+\theta, \theta<\lambda+1$, violates $S^{*}$ then it does not contain a part $>\lambda+1$ and hence all the parts will be among $1,2, \cdots, \lambda+1$. This implies that

$$
f_{1}+\cdots+f_{\lambda+1} \geq k=a \leftleftarrows a-1
$$

and hence such a partition violates $S$. This completes the proof of (3).

PROOF OF (4). First part of (4) can be proved on the same lines of (3). The second part of (4) is the case $k=a$ of the Conjecture.

As in the proof of (3) we can show that every partition in $P_{B}^{\prime}$ has an associate in $P_{A}^{\prime}$ except and this proves (4).

$$
\left(a-\frac{\lambda}{2}+1\right)(\lambda+1)
$$

CASE 2. Let $\lambda$ be odd.

PROOF OF (5). We prove (5) by establishing the following stronger result

$$
P_{B_{\lambda, k, a}}(n)=P_{D^{(n)}=P_{A_{\lambda, k, a}}(n) \text { for } n \leq \lambda .}
$$

From the definitions of $A_{\lambda, k, a}(n)$ and $B_{\lambda, k, a}(n)$ it is clear that $P_{A_{\lambda, k, a}}(n)=P_{D^{(n)}}$ and that $P_{B}(n) \subset P_{D^{(n)}}$. On the other hand, if $\pi \in P_{D^{(n)}}$ then $f_{i} \leq 1$ for $i=1,2, \cdots, \lambda$ and $f_{\lambda+1}=0$ as $n \leq \lambda$. Also 
and

$$
f_{\frac{\lambda+1}{2}}+\cdots+f_{\lambda} \leq 1
$$

$$
f_{1}+\cdots+f_{\lambda}=f_{1}+\cdots+f_{\frac{\lambda-1}{2}}+f_{\frac{\lambda+1}{2}}+\cdots+f_{\lambda} \leq \frac{\lambda-1}{2}+1=\frac{\lambda+1}{2}
$$

But $f_{1}+\cdots+f_{\lambda}=\frac{\lambda+1}{2}$ implies that the number being partitioned is $\geq 1+2+\cdots+\frac{\lambda-1}{2}+\frac{\lambda+1}{2}>\lambda$ Thus $f_{1}+\cdots+f_{\lambda} \leq \frac{\lambda-1}{2} \leq a-1$ since $\frac{\lambda-1}{2}<a$. Consider

$$
f_{2}+\cdots+f_{\lambda-1} \leq f_{2}+\cdots+f_{\frac{\lambda-1}{2}}+1 \leq\left(\frac{\lambda-1}{2}-1\right)+1=\frac{\lambda-1}{2}
$$

As before if $f_{2}+\cdots+\frac{f_{-1}}{2}=\frac{\lambda-1}{2}$ then the number being partitioned $\geq 2+3+\cdots+\frac{\lambda-1}{2}>\lambda$ and hence $f_{2}+\cdots+f_{\lambda-1} \leq \frac{\lambda-1}{2}-1 \leq a-2$ since $\frac{\lambda-1}{2}<a$. Proceeding like this we arrive at $\frac{f_{\lambda+1}}{2} \leq 1$ as $n \leq \lambda$ from which we obtain $f_{\frac{\lambda+1}{2}} \leq a-\frac{\lambda+1}{2}$.

For $x \in P_{D^{(n)}}$ and $n \leq \lambda$ the condition on $b$ 's is satisfied since no partition of $n$ has more than

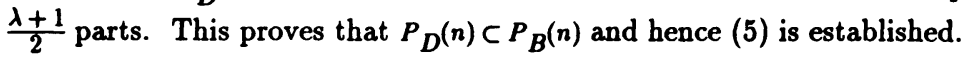

PROOF OF (6). From the definitions of $A_{\lambda, k, a}(n)$ and $B_{\lambda, k, a}(n)$ it is clear that

$$
P_{A}^{\prime}(\lambda+1)=\left\{\begin{array}{l}
\frac{\lambda+3}{2}+\frac{\lambda-1}{2} \text { when } a=\frac{\lambda+1}{2} \\
\frac{\lambda+1}{2}+\frac{\lambda+1}{2} \text { when } a>\frac{\lambda+1}{2}
\end{array}\right.
$$

and $P_{B}^{\prime}(\lambda+1)=\{(\lambda+1)\}$

PROOF OF (7). For $n=(\lambda+1+\theta), \quad \theta<\frac{\lambda+1}{2}$

$$
P_{A}^{\prime}(n)=\left\{\begin{array}{cc}
\frac{\lambda+3}{2}+\frac{\lambda-1}{2}+\pi: \pi \in P_{D^{(}}(\theta) & \text { with parts }<\frac{\lambda-1}{2}, \theta<\frac{\lambda-1}{2}, a=\frac{\lambda+1}{2} \\
\frac{\lambda+5}{2}+\frac{\lambda-1}{2}+\frac{\lambda-3}{2}, \frac{\lambda+3}{2}+\frac{\lambda-1}{2}+\pi: \pi \in P_{D^{\prime}}(\theta) \text { with parts }<\frac{\lambda-1}{2}, \theta=\frac{\lambda-1}{2}, a=\frac{\lambda+1}{2} \\
\frac{\lambda+1}{2}+\frac{\lambda+1}{2}+\pi: \pi \in P_{D}(\theta) \quad \text { and } a>\frac{\lambda+1}{2} \\
P_{B}^{\prime}(n)=\left\{(\lambda+1)+\pi: \pi \in P_{D}(\theta)\right\}
\end{array}\right.
$$

PROOF of (8). Clearly

$$
\begin{aligned}
& P_{A}^{\prime}(n)=\left\{\begin{array}{l}
\frac{\lambda+5}{2}+\frac{\lambda-1}{2}+\pi: \pi \in P_{D^{D}}\left(\frac{\lambda-1}{2}\right) \quad \text { with parts }<\frac{\lambda-1}{2} \text { and } a=\frac{\lambda+1}{2} \\
\frac{\lambda+3}{2}+\frac{\lambda+1}{2}+\frac{\lambda-1}{2}, \frac{\lambda+1}{2}+\frac{\lambda+1}{2}+\pi: \pi \in P_{D^{(}}\left(\frac{\lambda+1}{2}\right) \text { and } a=\frac{\lambda+3}{2} \\
\frac{\lambda+1}{2}+\frac{\lambda+1}{2}+\pi: \pi \in P_{D}\left(\frac{\lambda+1}{2}\right) \text { and } a>\frac{\lambda+3}{2}
\end{array}\right. \\
& P_{B}^{\prime}(n)=\left\{\begin{array}{l}
\frac{3}{2}(\lambda+1),(\lambda+1)+\pi: \pi \in P_{D}\left(\frac{\lambda+1}{2}\right) \quad \text { with parts }<\frac{\lambda+1}{2} \text { and } a=\frac{\lambda+1}{2} \\
\frac{3}{2}(\lambda+1),(\lambda+1)+\pi: \pi \in P_{D}\left(\frac{\lambda+1}{2}\right) \text { and } a=\frac{\lambda+3}{2} \\
(\lambda+1)+\pi: \pi \in P_{D}\left(\frac{\lambda+1}{2}\right) \text { and } a>\frac{\lambda+3}{2}
\end{array}\right.
\end{aligned}
$$


When $a=\frac{\lambda+1}{2}=k$, the $n$ in the conjecture becomes $\frac{3}{2}(\lambda+1)$ and $\frac{3}{2}(\lambda+1) \in P_{B}^{\prime}$ has no associate in $P_{A}^{\prime}$ and this establishes the conjecture when $k=a=\frac{\lambda+1}{2}$.

PROOF OF (9). Let $n=(2 a-\lambda+1)\left(\frac{\lambda+1}{2}\right)+\theta, \theta<\frac{\lambda+1}{2}$. Now $\pi \in P_{A}^{\prime}(n)$ implies $\pi$ violates one of the conditions $S_{1}, \cdots, S_{\frac{\lambda+1}{2}}, S, S^{*}, S^{* *}$ where $S^{* *}$ is the condition "no parts $\neq 0(\bmod \lambda+1)$ are repeated". A proof similar to that of Step $\frac{\lambda}{2}+2$ of even $\lambda$ will show that partitions violating $S^{*}$ will also violate $S_{1}$. Since no part is $\equiv \lambda+1(\bmod 2 \lambda+2)$ for partitions enumerated by $A_{\lambda, k, a}(n)$ we have $f_{\lambda+1}=0$ and hence $S$ reduces to $S_{1}$. In the following steps 1 to $\frac{\lambda+3}{2}$, we enumerate the partitions in $P_{A}$ violating $S_{\frac{\lambda+1}{2}}, \cdots, S_{1}, S^{* *}$ and also give the bijection of $P_{A}^{\prime}(n)$ onto $P_{B}^{\prime}(n)$.

STEP 1. Consider $\frac{S_{\frac{\lambda+1}{2}}}{2}: \frac{f_{\lambda+1}}{2} \leq 1 \leq\left(a-\frac{\lambda+1}{2}\right)$. Clearly there are no partitions in $P_{A}$ violating $S_{\frac{\lambda+1}{2}}$ for $a-\frac{\lambda+1}{2} \geq 1$. Since $\frac{\lambda+1}{2}$ is not a part of partitions enumerated by both $A_{\lambda, k, a}(n)$ and $B_{\lambda, k, a}(n)$ when $a=\frac{\lambda+1}{2}$ it follows that there are no partitions violating $S_{\frac{\lambda+1}{2}}$ when $a=\frac{\lambda+1}{2}$ also.

STEP 2. Consider $S_{\frac{\lambda-1}{2}}: f_{\frac{\lambda-1}{2}}+f_{\frac{\lambda+1}{2}}+f_{\frac{\lambda+3}{2}} \leq 3 \leq a-\frac{\lambda-1}{2}$

For $a \geq \frac{\lambda+5}{2}$ there are no partitions in $P_{A}$ violating $S_{\frac{\lambda-1}{1}}$. If $a=\frac{\lambda+1}{2}$, then $n=(\lambda+1)+\theta$, $\Theta<\frac{\lambda+1}{2}$ and the set of partitions violating $S_{\frac{\lambda-1}{2}}$ is $\left\{\frac{\lambda+3}{2}+\frac{\frac{\lambda-1}{2}}{2}+\pi: \pi \in P_{D}(\Theta)\right\}$ For each partition in the above set we associate $(\lambda+1)+\pi$ in $P_{B}^{\prime}$. Let $a=\frac{\lambda+3}{2}$. Then $n=2(\lambda+1)+\Theta, \Theta<\frac{\lambda+1}{2}$ and the set of partitions violating $S_{\frac{\lambda-1}{2}}$ is

$$
\begin{array}{r}
\left\{\frac{\lambda+3}{2}+\frac{\lambda+1}{2}+\frac{\lambda-1}{2}+\pi: \pi \in P_{D}\left(\frac{\lambda+1}{2}+\theta\right) \quad \text { with parts }<\frac{\lambda-1}{2}\right\} \\
\cup\left\{\left(\frac{\lambda+1}{2}+\Theta^{\prime}\right)+\frac{\lambda+3}{2}+\frac{\lambda+1}{2}+\frac{\lambda-1}{2}+\pi: \pi \in P_{D}\left(\theta-\Theta^{\prime}\right), 2 \leq \Theta^{\prime} \leq \frac{\lambda-1}{2}\right\}
\end{array}
$$

We associate $\frac{3}{2}(\lambda+1)+\pi \in P_{B}^{\prime}$ for every partition in the first set while for a partition in the second set we associate $\frac{3}{2}(\lambda+1)+\left(\frac{\lambda+1}{2}+\Theta^{\prime}\right)+\pi$ in $P_{B}^{\prime}$.

Proceeding like this we arrive at the following step.

STEP $\frac{\lambda+1}{2}$. Consider $S_{1}: f_{1}+\cdots+f_{\lambda} \leq a-1$. By the definition of $A_{\lambda, k, a}(n), f_{i} \leq 1$ for all $i=1, \cdots, \lambda$ except for $i=\frac{\lambda+1}{2}$. But $1 \leq f_{\frac{\lambda+1}{2}} \leq 2 a-\lambda+1$. The case $\frac{f^{\lambda+1}}{2}>1$ will be considered in step $\frac{\lambda+3}{2}$. Hence let us now assume $\frac{f^{\frac{\lambda+1}{2}}}{2} \leq 1$.

In this case $f_{1}+\cdots+f_{\lambda} \leq \lambda$. If $f_{1}+\cdots+f_{\lambda}=\lambda$, then $1+2+\cdots+\lambda=\frac{\lambda}{2}(\lambda+1)=\frac{\lambda-1}{2}(\lambda+1)+\frac{\lambda+1}{2}$ $\geq\left(a-\frac{\lambda-1}{2}\right)(\lambda+1)+\frac{\lambda+1}{2}>n$. Thus there are no partitions violating $S_{1}$ in $P_{A}^{\prime}$. Let $f_{1}+\cdots+f_{\lambda}=\lambda-1$ and let the deleted part be $x$. Consider

$$
\begin{aligned}
& 1+2+\cdots+(x-1)+(x+1)+\cdots+(\lambda-1)+\lambda \\
& =(\lambda-2)\left(\frac{\lambda+1}{2}\right)+(\lambda+1-x) \text { where } 1 \leq(\lambda+1-x) \leq \lambda .
\end{aligned}
$$

If $2 a-\lambda+1<\lambda-2$ then (19) is $>n$ and hence there will be no partitions of $n$ violating $S_{1}$. Clearly $2 a-\lambda+1 \neq \lambda-2$. When $2 a-\lambda+1>\lambda-2$ the only partition of $n$ violating $S_{1}$ is

$$
\lambda+(\lambda-1)+\cdots+(x+1)+(x-1)+\cdots+2+1 \quad \text { with } \frac{\lambda+1}{2}-x=\Theta
$$

for which we associate the following partition in $P_{B}^{\prime}$ 


$$
\underbrace{(\lambda+1)+\cdots+(\lambda+1)}_{\frac{\lambda-3}{2} \text { times }}+\left(\frac{\lambda+1}{2}+\theta\right)+\frac{\lambda+1}{2}
$$

More generally, let $f_{1}+\cdots+f_{\lambda}=\lambda-y \quad(1 \leq y \leq \lambda-a)$ and let $x_{1}, \cdots, x_{y}$ with $1 \leq x_{1}<x_{2}<\cdots<x_{y} \leq \lambda$ be the parts deleted among $1,2, \cdots, \lambda$. Then

$$
\begin{aligned}
& \lambda+(\lambda-1)+\cdots+\left(x_{y}+1\right)+\left(x_{y}-1\right)+\cdots+\left(x_{1}+1\right)+\left(x_{1}-1\right)+\cdots+2+1 \\
& =(\lambda-2 y)\left(\frac{\lambda+1}{2}\right)+\left(\lambda+1-x_{1}\right)+\cdots+\left(\lambda+1-x_{y}\right) .
\end{aligned}
$$

If $2 a-\lambda+1<\lambda-2 y$ then $(20)$ is $>n$ and hence there are no partitions of $n$ violating $s_{1}$. Also $2 a-\lambda+1 \neq \lambda-2 y$. Let $2 a-\lambda+1>\lambda-2 y$. Then $\lambda-2 y+1 \leq 2 a-\lambda+1 \leq \lambda-1$. If $2 a-\lambda+1>\lambda-2 y+1$ then $f_{1}+\cdots+f_{\lambda}=\lambda-y \leq a-1$ and hence there will be no partitions of $n$ violating $S_{1}$. If $2 a-\lambda+1=\lambda-2 y+1$ and if $\left(\lambda+1-x_{1}\right)+\cdots+\left(\lambda+1-x_{y}\right)>\frac{\lambda+1}{2}+\theta$ then (20) is $>n$. On the other hand, if $\left(\lambda+1-x_{1}\right)+\cdots+\left(\lambda+1-x_{y}\right)<\frac{\lambda+1}{2}+\theta$ then also there are no partitions of $n$ violating $S_{1}$ since in this case parts have to be repeated. Since $\frac{\lambda+1}{2}+\theta<\lambda+1$ we note that $\left(\lambda+1-x_{1}\right)+\cdots+\left(\lambda+1-x_{y}\right)=\frac{\lambda+1}{2}+\Theta$ is possible only if

(a) $x_{1}<\frac{\lambda+1}{2}, x_{2}=\frac{\lambda+1}{2}$ and $x_{i}>\frac{\lambda+1}{2}$ for $i=3, \cdots y$

(b) $x_{1}<\frac{\lambda+1}{2}$ and $x_{i}>\frac{\lambda+1}{2}$ for $i=2, \cdots, y$

(c) $x_{1}=\frac{\lambda+1}{2}$ and $x_{i}>\frac{\lambda+1}{2}$ for $i=2, \cdots, y$

(d) $x_{i}>\frac{\lambda+1}{2}$ for $i=1, \cdots, y$

In each of the cases (a)-(d) the partition on the left hand side of (20) violates $S_{1}$ for which we respectively associate the following partitions in $P_{B}^{\prime}$.

$$
\begin{aligned}
& \underbrace{(\lambda+1)+\cdots+(\lambda+1)}_{\left(\frac{\lambda-2 y+1}{2}\right) \text { times }}+\left(\lambda+1-x_{1}\right)+\left(\lambda+1-x_{3}\right)+\cdots+\left(\lambda+1-x_{y}\right) \\
& \underbrace{(\lambda+1)+\cdots+(\lambda+1)}_{\left(\frac{\lambda-2 y-1}{2}\right) \text { times }}+\left(\lambda+1-x_{1}\right)+\frac{\lambda+1}{2}+\left(\lambda+1-x_{2}\right)+\cdots+\left(\lambda+1-x_{y}\right) \\
& \underbrace{(\lambda+1)+\cdots+(\lambda+1)}_{\left(\frac{\lambda-2 y+1}{2}\right) \text { times }}+\left(\lambda+1-x_{2}\right)+\cdots+\left(\lambda+1-x_{y}\right) \\
& \underbrace{(\lambda+1)+\cdots+(\lambda+1)}_{\left(\frac{\lambda-2 y-1}{2}\right) \text { times }}+\frac{\lambda+1}{2}+\left(\lambda+1-x_{1}\right)+\cdots+\left(\lambda+1-x_{y}\right)
\end{aligned}
$$

STEP $\frac{\lambda+3}{2}$. Consider $S^{* *}$ : 'no parts $\not \equiv 0(\bmod \lambda+1)$ are repeated'. This implies that $\frac{f_{\lambda+1}}{2} \geq 2$. When $a=\frac{\lambda+1}{2}$ there are no partitions violating $S^{* *}$ since $\frac{\lambda+1}{2}$ is not a part for partitions enumerated by both $A_{\lambda, k, a}(n)$ and $B_{\lambda, k, a}(n)$.

Let $a=\frac{\lambda+3}{2}$. Then $n=2(\lambda+1)+\theta, \Theta<\frac{\lambda+1}{2}$. The set of partitions in $P_{A}^{\prime}$ violating $S^{* *}$ is

$$
\begin{aligned}
& \left\{\frac{\lambda+1}{2}+\frac{\lambda+1}{2}+\frac{\lambda+1}{2}+\frac{\lambda+1}{2}+\pi: \pi \in P_{D}(\Theta)\right\} \\
& \cup\left\{\frac{\lambda+1}{2}+\frac{\lambda+1}{2}+\frac{\lambda+1}{2}+\pi: \pi \in P_{D}\left(\frac{\lambda+1}{2}+\Theta\right) \quad \text { with parts }<\frac{\lambda+1}{2}\right\} \\
& \cup\left\{\left(\frac{\lambda+1}{2}+\Theta^{\prime}\right)+\frac{\lambda+1}{2}+\frac{\lambda+1}{2}+\frac{\lambda+1}{2}+\pi: \pi \in P_{D^{\prime}}\left(\Theta-\Theta^{\prime}\right), 1 \leq \Theta^{\prime} \leq \frac{\lambda-1}{2}\right\} \\
& \left.\cup\left\{\frac{\lambda+1}{2}+\frac{\lambda+1}{2}+\pi: \pi \in P_{D^{(\lambda+1}}+\Theta\right) \quad \text { with parts }<\frac{\lambda+1}{2}\right\}
\end{aligned}
$$




$$
\begin{aligned}
& \cup\left\{\left(\frac{\lambda+1}{2}+\Theta^{\prime}\right)+\frac{\lambda+1}{2}+\frac{\lambda+1}{2}+\pi: \pi \in P_{D^{(}}\left(\frac{\lambda+1}{2}+\Theta-\Theta^{\prime}\right) \text { parts }<\frac{\lambda+1}{2}, 1 \leq \Theta^{\prime} \leq \frac{\lambda-1}{2}\right\} \\
& \cup\left\{\left(\frac{\lambda+1}{2}+\Theta^{\prime \prime}\right)+\left(\frac{\lambda+1}{2}+\Theta^{\prime}\right)+\frac{\lambda+1}{2}+\frac{\lambda+1}{2}+\pi: \pi \in P_{D^{\prime}}\left(\Theta-\Theta^{\prime}-\Theta^{\prime \prime}\right), 1 \leq \Theta^{\prime}<\Theta^{\prime \prime} \leq \frac{\lambda-1}{2}\right\}
\end{aligned}
$$

For each of the above sets of partitions in $P_{A}^{\prime}$ we respectively associate the following sets of partitions in $P_{B}^{\prime}$.

$$
\begin{aligned}
& \left\{\frac{3}{2}(\lambda+1)+\frac{\lambda+1}{2}+\pi: \pi \in P_{D}(\Theta)\right\} \\
& \cup\left\{(\lambda+1)+\left(\frac{\lambda+1}{2}\right)+\pi: \pi \in P_{D^{(}}\left(\frac{\lambda+1}{2}+\theta\right) \text { parts }<\frac{\lambda+1}{2}\right\} \\
& U\left\{(\lambda+1)+\left(\frac{\lambda+1}{2}+\Theta^{\prime}\right)+\frac{\lambda+1}{2}+\pi: \pi \in P_{D^{(}}\left(\theta-\theta^{\prime}\right), 1 \leq \Theta^{\prime} \leq \frac{\lambda-1}{2}\right\} \\
& \left.\cup\left\{(\lambda+1)+\pi: \pi \in P_{D^{(\lambda+1}}+\theta\right) \text { parts }<\frac{\lambda+1}{2}\right\} \\
& \cup\left\{(\lambda+1)+\left(\frac{\lambda+1}{2}+\Theta^{\prime}\right)+\pi: \pi \in P_{D^{(}}\left(\frac{\lambda+1}{2}+\theta-\Theta^{\prime}\right) \text { parts }<\frac{\lambda+1}{2}, 1 \leq \Theta^{\prime} \leq \frac{\lambda-1}{2}\right\} \\
& \cup\left\{(\lambda+1)+\left(\frac{\lambda+1}{2}+\Theta^{\prime \prime}\right)+\left(\frac{\lambda+1}{2}+\Theta^{\prime}\right)+\pi: \pi \in P_{D^{(}}\left(\theta-\Theta^{\prime}-\Theta^{\prime \prime}\right), 1 \leq \Theta^{\prime}<\Theta^{\prime \prime} \leq \frac{\lambda-1}{2}\right\}
\end{aligned}
$$

For any given ' $a$ ' we can similarly enumerate the partitions in $P_{A}^{\prime}$ violating $S^{* *}$ and also can obtain the bijection of $P_{A}^{\prime}$ onto $P_{B}^{\prime}$. The proof of (9) now follows from Steps 1 to $\frac{\lambda+3}{2}$.

PROOF OF (10). The first part of (10) follows on a line similar to the proof of (9). The second part of (10) is the case $k=a$ of the conjecture. As in the proof of (9) we can show that every partition in $P_{B}^{\prime}$ has an associate in $P_{A}^{\prime}$ except $(2 a-\lambda+2)\left(\frac{\lambda+1}{2}\right)$ and this proves (10).

We now consider some numerical examples.

EXAMPLE 1. Let $\lambda=4, k=3=a, n=\left(\begin{array}{c}k+\lambda-a+1 \\ 2\end{array}\right)+(k-\lambda+1)(\lambda+1)=10$.

\section{TABLE 1}

n

$$
P_{A_{4,3,3}}{ }^{(n)}
$$

$$
\{1\}
$$

$3 \quad\{3,2+1\}$

$4\{4,3+1\}$

$5 \quad\{4+1\} \cup\{3+2\}$

$6 \quad\{6,4+2\} \cup\{3+2+1\}$

$7 \quad\{7,6+1,4+3\} \cup\{4+2+1\}$

$8 \quad\{8,7+1,6+2\} \cup\{4+3+1\}$

$9 \quad\{9,8+1,7+2,6+3,6+2+1\} \cup\{4+3+2\}$

$10\{9+1,8+2,7+3,7+2+1,6+4,6+3+1\}$

$\cup\{4+3+2+1\}$
$P_{B_{4,3,3}}{ }^{(n)}$

$\{1\}$

$\{2\}$

$\{3,2+1\}$

$\{4,3+1\}$

$\{4+1\} \cup\{5\}$

$\{6,4+2\} \cup\{5+1\}$

$\{7,6+1,4+3\} \cup\{5+2\}$

$\{8,7+1,6+2\} \cup\{5+3\}$

$\{9,8+1,7+2,6+3,6+2+1\} \cup\{5+4\}$

$\{9+1,8+2,7+3,7+2+1,6+4,6+3+1\}$

$\cup\{10,5+5\}$

According to the proofs of (1)-(4), we have
(a) $\quad P_{A_{4,3,3}}{ }^{(n)}=P_{B_{4,3,3}}{ }^{(n)} \quad$ for $n \leq 4$
(b) $\quad P_{A_{4,3,3}}^{\prime}(5)=\{3+2\}, \quad P_{B_{4,3,3}}^{\prime}{ }^{(5)}=\{5\}$ 
(c) The partitions enumerated by $A_{4,3,3}(n)$ for $n=6,7,8,9$ violating $S_{2}$ according to Step 1 in the proof of (3) are

$$
\{3+2+1\} \cup\{4+3+2\}
$$

for which their associates in $P_{B}^{\prime}$ are

$$
\{5+1\} \cup\{5+4\}
$$

(d) The partitions enumerated by $A_{4,3,3}(n)$ for $n=6,7,8,9$ violating $S_{1}$ as proved in Step 2 are

$$
\{4+2+1\} \cup\{4+3+1\}
$$

for which the corresponding partitions in $P_{B}^{\prime}$ are

$$
\{5+2\} \cup\{5+3\}
$$

(e) The partitions enumerated by $A_{4,3,3}(n)$ for $n=6,7,8,9$ violating $S$ also violate $S_{1}$ or $S_{2}$. have.

(f) The partition $10=2 \times(4+1) \in P_{B_{4,3,3}^{\prime}}(10)$ has no associate in $P_{A}^{\prime}$ while all other partitions

From Table 1 it is clear that (a)-(f) are indeed true.

EXAMPLE 2. Let $\lambda=5, k=a=3, n=\left(\begin{array}{c}k+\lambda-a+1 \\ 2\end{array}\right)+(k-\lambda+1)(\lambda+1)=9$.

\section{TABLE 2}

n

$$
P_{A_{5,3,3}}^{(n)}
$$

\{1\}

\{2\}

$$
\{2+1\}
$$

\{4\}

$\{5,4+1\}$

$\{5+1\} \cup\{4+2\}$

$7 \quad\{7,5+2\} \cup\{4+2+1\}$

$8 \quad\{8,7+1\} \cup\{5+2+1\}$

$9 \quad\{8+1,7+2,5+4\}$

$$
P_{B_{5,3,3}}{ }^{(n)}
$$

\{1\}

\{2\}

$\{2+1\}$

\{4\}

$\{5,4+1\}$

$\{5+1\} \cup\{6\}$

$\{7,5+2\} \cup\{6+1\}$

$\{8,7+1\} \cup\{6+2\}$

$\{8+1,7+2,5+4\} \cup\{9\}$

From the proofs of (5)-(8) we have the following:

(g) $\quad P_{A_{5,3,3}}{ }^{(n)=P_{B_{5,3,3}}}{ }^{(n)} \quad$ for $n \leq 5$

(h) $P_{A_{5,3,3}}^{\prime(6)}=\{4+2\} \quad P_{B_{5,3,3}}^{\prime(6)}=\{6\}$

(i) $\quad P_{A_{5,3,3}}^{\prime}(7)=\{4+2+1\} \quad P_{B_{5,3,3}}^{\prime}(7)=\{6+1\}$

(j) $\quad P_{A_{5,3,3}}^{\prime}(8)=\{5+2+1\} \quad P_{B_{5,3,3}}^{\prime}(8)=\{6+2\}$

(k) The partition $(2 \times 3-5+2)\left(\frac{5+1}{2}\right)=9$ in $P_{B_{5,3,3}}^{\prime}(9)$ has no associate in $P_{A_{5,3,3}}^{\prime}$ (9) while all others have.

From Table 2 it is evident that the results $(\mathrm{g})-(\mathrm{k})$ are true. 
ACKNOWLEDGEMENT. The authors are indebted and grateful to Professor George E. Andrews for suggesting the problem and for his constant help during the course of the work.

\section{REFERENCES}

1. ANDREWS, G.E., On the general Rogers-Ramanujan theorem, Mem. Amer. Math. Soc. No. 152 (1974), 1-86. 


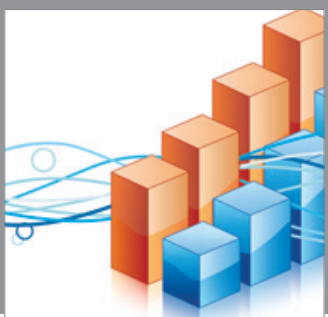

Advances in

Operations Research

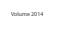

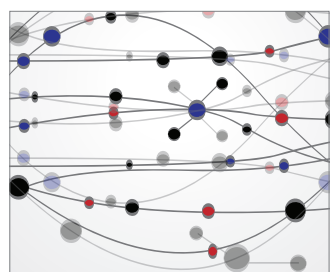

\section{The Scientific} World Journal
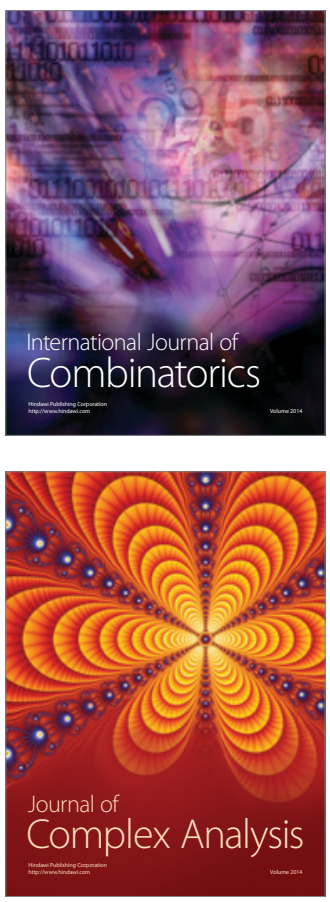

International Journal of

Mathematics and

Mathematical

Sciences
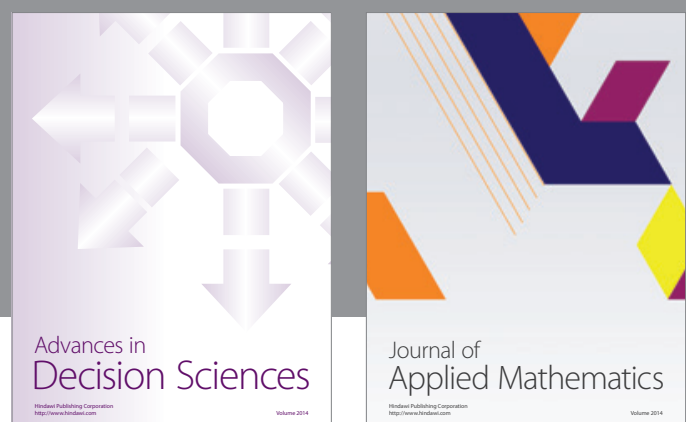

Journal of

Applied Mathematics
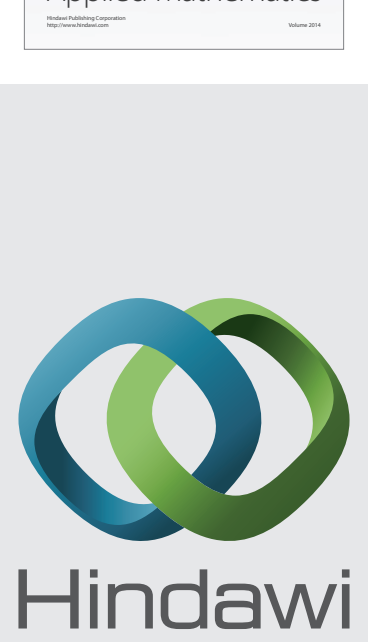

Submit your manuscripts at http://www.hindawi.com
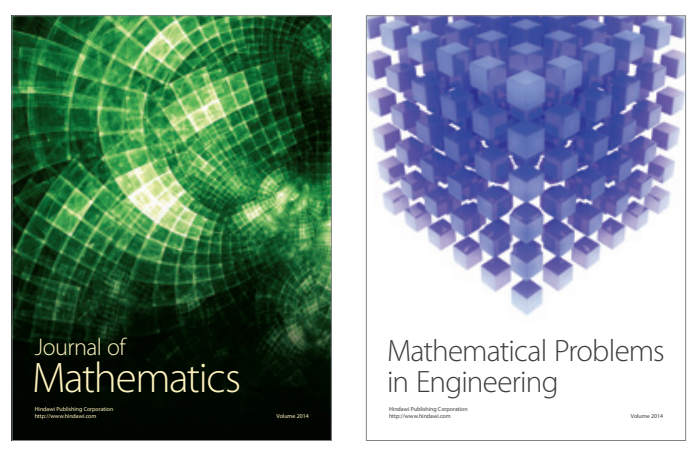

Mathematical Problems in Engineering
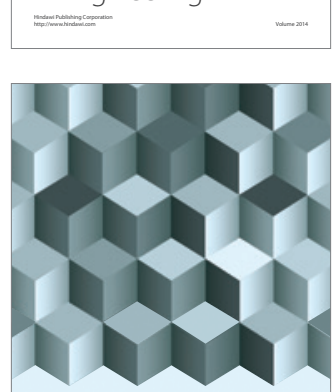

Journal of

Function Spaces
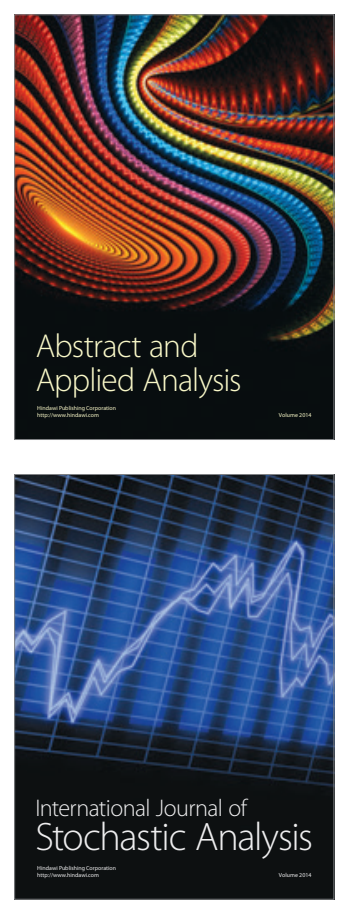

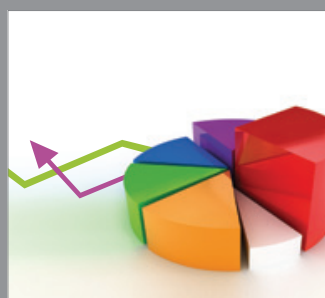

ournal of

Probability and Statistics

Promensencen
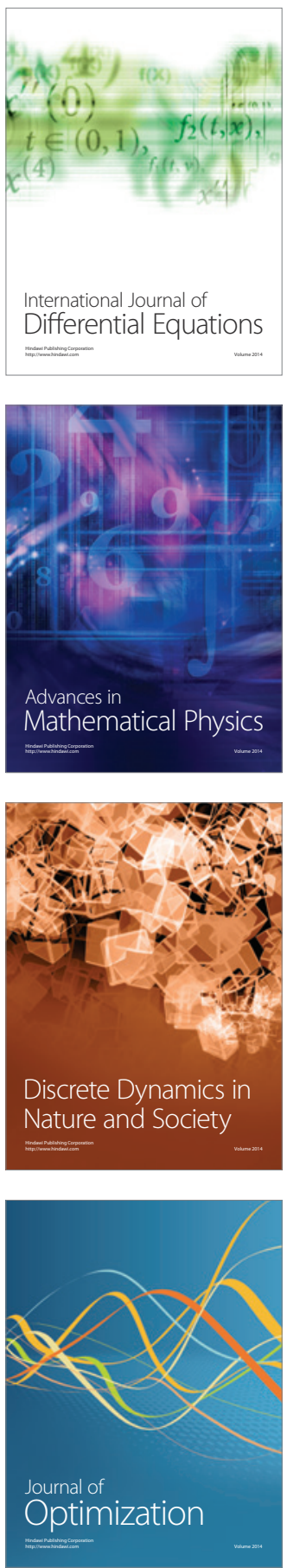